\title{
Design and Fabrication of a High Aspect Ratio Cable for a High Gradient Quadrupole Magnet
}

\author{
R.M. Scanlan, A.D. McInturff, C.E. Taylor, S. Caspi, D. Dell'Orco, and H. Higley \\ Lawrence Berkeley National Laboratory \\ S. Gourlay, R. Bossert, J. Brandt, and A.V. Zlobin \\ Fermi National Accelerator Laboratory
}

\begin{abstract}
The Large Hadron Collider interaction regions require quadrupoles with a $\mathbf{7 0}$ $\mathrm{mm}$ diameter bore, a gradient of $250 \mathrm{~T} / \mathrm{m}$, and good cooling so that the magnets can operate in a high radiation background without quenching. In order to meet these stringent requirements, a two-layer magnet with a high aspect ratio cable has been designed. This cable utilizes the SSC inner and outer layer strands, which have been optimized and are available in large quantities. The initial design parameters for both cables are $15.2 \mathrm{~mm}$ width; the inner cable has 38 strands of $0.8 \mathrm{~mm}$ diam wire and a keystone angle of 0.99 deg. The outer cable has 46 strands of $0.65 \mathrm{~mm}$ diam wire and a keystone angle of $0.69 \mathrm{deg}$. These cables have been fabricated and then subjected to a number of tests to insure their performance in the quadrupole. These test results, including model coil winding studies, electrical property measurements, and mechanical property measurements will be presented.
\end{abstract}

\section{INTRODUCTION}

During the past year, significant progress has been made in developing a plan for a U.S. contribution to the Large Hadron Collider (LHC) project being constructed at CERN in Geneva, Switzerland. In anticipation of this U.S. participation in LHC, a consortium of Brookhaven National Lab, Fermilab, and Lawrence Berkeley National Lab was formed to identify areas where the U.S. can make significant technical contributions. One of these areas is the high gradient quadrupoles for the interaction regions. Several different design approaches for these magnets were studied at a workshop involving experts from all three labs, held at Fermilab in July, 1995. The results of this design study and the details of the design are presented in a companion paper at this conference (1), so only a brief summary will be presented here. The design chosen for further optimization and development is a two layer, $\cos 2$ theta cross section with a $70 \mathrm{~mm}$ bore. This design was chosen in preference to a 4layer geometry for the following: (1) quench protection is simplified due to a lower inductance and also because quench heaters can be placed in close proximity to both layers, (2) heat removal should be more efficient with fewer layers and

Manuscript received August 27, 1996

This work was supported by the Director, Office of Energy Research, Office of High Energy and Nuclear Physics, High Energy Physics Division, U.S. Department of Energy, under Contract No. DE-AC03-76SF0098. hence fewer insulation layers to impede heat flow, and (3) this design takes advantage of a good experience base as well as existing tooling. However, a major challenge in this magnet design is achieving the required gradient with only two layers--this requires high aspect ratio cables for the two layers. In addition, it was recognized that considerable development time and expense could be saved if the existing SSC strand could be utilized. This paper describes the fabrication and testing of the high aspect ratio cable designed to meet the requirements of this high gradient quadrupole.

\section{CABLE DESIGN}

During the past 10 years, the Experimental Cabling Facility at LBNL has been used to fabricate a series of Rutherford cables in which the basic parameters were examined in a systematic way. This work (2) has led to a set of empirical guidelines that can be used to set the parameters of a new cable design. These guidelines have been incorporated into a design program (3) that allows the cable or magnet designer to find, for example, the maximum safe keystone angle and compaction for a cable of given width and thickness. Using these guidelines, optimized cable parameters can be determined for an inner layer cable and an outer layer cable using existing SSC strands. However, for this magnet design, an additional constraint was applied, i.e., that both cables should have the same width in order to facilitate a splice between inner and outer cables within the outer coil. In the case of fixed wire diameters (existing SSC wire) as well, this represents an overconstrained problem so that the compaction could be optimized for one cable only. In addition, these cables are well beyond the range of cable production experience in terms of aspect ratio (see Table I), so a cable fabrication and testing program was initiated.

TABLE I

Parameters for various Rutherford type cables

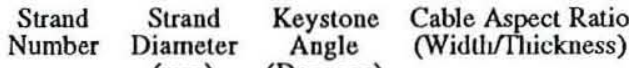

Cable Number $\underset{(\mathrm{mm})}{\text { Diameter }} \underset{\text { (Degrees) }}{\text { Angle }}$

$\begin{array}{lllll}\text { SSC Inner } & 30 & 0.808 & 1.2 & 8.5\end{array}$

$\begin{array}{lllll}\text { SSC Outer } & 36 & 0.648 & 1.01 & 10.1\end{array}$

$\begin{array}{lllll}\text { LHC Inner } & 28 & 1.065 & 1.30 & 7.9\end{array}$

$\begin{array}{lllll}\text { LHC Outer } & 36 & 0.825 & 1.00 & 10.2\end{array}$

$\begin{array}{lllll}\text { FNAL LBQ } & 36 & 0.528 & 1.06 & 10.9\end{array}$

$\begin{array}{lllll}\text { LHC HGQ } & 38 & 0.808 & 0.99 & 10.6\end{array}$

Inner

(this design)

LHC HGQ

46

0.648

0.69

13.4

(this design) 


\section{CABLE FABRICATION}

A series of cables were fabricated at the Experimental Cabling Facility at LBNL. In order to insure that variations in strand properties did not confuse the results, strand from a single manufacturer (Intermagnetics General Corp.) was used for all inner cables and likewise strand from Furukawa Electric Corp. was used in all outer cables. The cabling machine has the following features that are useful for cabling development work: up to 60 strand capacity; adjustable strand tension and adjustable strand twisting; powered Turkshead; cable measuring machine for precise dimensional measurements. In addition, this cabling machine has been used to fabricate production lengths of cable for the SSC program and the low beta quadrupole program at FNAL.

Using the cable optimization algorithm, the target cable parameters listed in Table I were chosen as a starting point. As stated above, the requirement of equal cable widths for both inner and outer cables, together with the fixed strand diameters, meant that the compaction could not be optimized for both cables. The initial cable parameters were chosen to yield an inner cable that was somewhat overcompacted (from the standpoint of expected critical current degradation) and an outer cable that was somewhat undercompacted (from the cable stability standpoint). Since the cable parameters were not optimum, a series of cables were fabricated in which the compaction was varied in two ways: the number of strands was reduced and the width of the cables was increased. One additional restriction associated with using the existing SSC wire is that both inner and outer wire are twisted in the same direction, and this means that for a minimum residual twist in the cables, both inner and outer cables should be made with the same lay direction. Since the mating inner and outer coils are wound in opposite directions, this means that the cable in one layer must be wound in the unfavorable direction (from the standpoint of stability against strand popping and a tendency toward decabling). In order to evaluate the effects of cable lay direction, samples were prepared with the opposite lay from strands with the same twist even though this leads to an undesirable residual twist in the cable. The parameters of the various cables are listed in Table II.

TABLE II

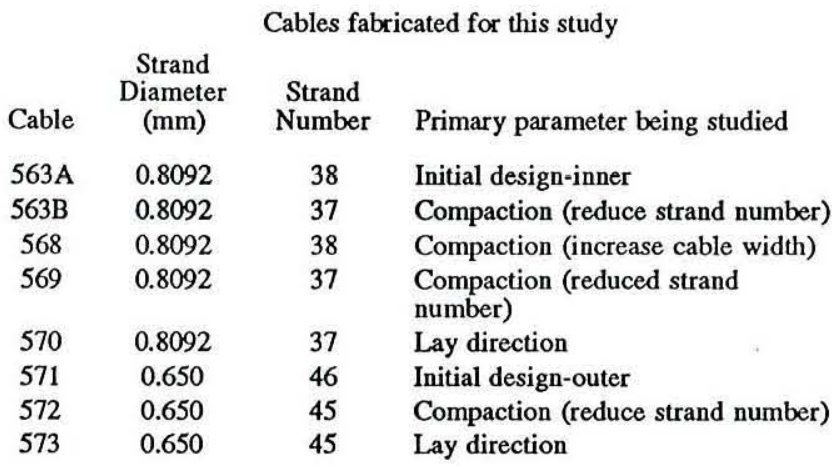

\section{CABLE ELECTRICAL MEASUREMENTS}

The critical current was determined for selected samples at BNL in their standard cable critical current test fixture (4). The critical current at $4.2 \mathrm{~K}$ was determined in the field range
6-7.5 $\mathrm{T}$ and the critical current densities were calculated at 7.0 T. These values, together with the relevant cable properties, are listed in Table III. Not all samples fabricated for this study were measured; the samples where only the lay direction was reversed were not measured, nor was the 45 strand outer cable, which was mechanically unstable and could not be mounted for the short sample critical current test.

TABLE III

\begin{tabular}{|c|c|c|c|c|c|c|}
\hline & & Measured & cable para & eters for thi & & \\
\hline & & ble Dimens & ions & Compaction & & \\
\hline & & & Keystone & (Strand Area & & \\
\hline Cable & $\begin{array}{l}\text { Width } \\
(\mathrm{mm})\end{array}$ & $\begin{array}{l}\text { Thickness } \\
\text { (mm) }\end{array}$ & $\begin{array}{l}\text { Angle } \\
\text { (Deg) }\end{array}$ & & $\begin{array}{c}\text { Jc (7T) } \\
\text { (A.mm2) }\end{array}$ & $\begin{array}{c}\text { Ic (7T) } \\
(A)\end{array}$ \\
\hline $563 \mathrm{~A}$ & 15.287 & 1.457 & 0.85 & 0.910 & 1153 & 9879 \\
\hline $563 \mathrm{~B}$ & 15.202 & 1.456 & 0.86 & 0.892 & 1682 & 14034 \\
\hline 568 & 15.386 & 1.458 & 1.07 & 0.904 & 1674 & 14234 \\
\hline 569 & 15.377 & 1.451 & 1.09 & 0.885 & 1742 & 14237 \\
\hline 571 & 15.380 & 1.150 & 0.69 & 0.898 & 1703 & 9354 \\
\hline
\end{tabular}

\section{COIL WINDING TESTS}

The cables listed in Table II were sent to FNAL for a series of coil winding tests. The cables were wound in the bare and insulated condition, in both the favorable and unfavorable winding direction, and with varying winding tension. In addition, several different shapes of the pole islands were used. However, the final pole shape, which will be produced by machining to the shape determined by the BEND (5) design program, has not been fabricated.

By using the power Turkshead (Fig. 1) to reduce the tension on the cable, all versions of these high aspect ratio cables could be made without difficulty.

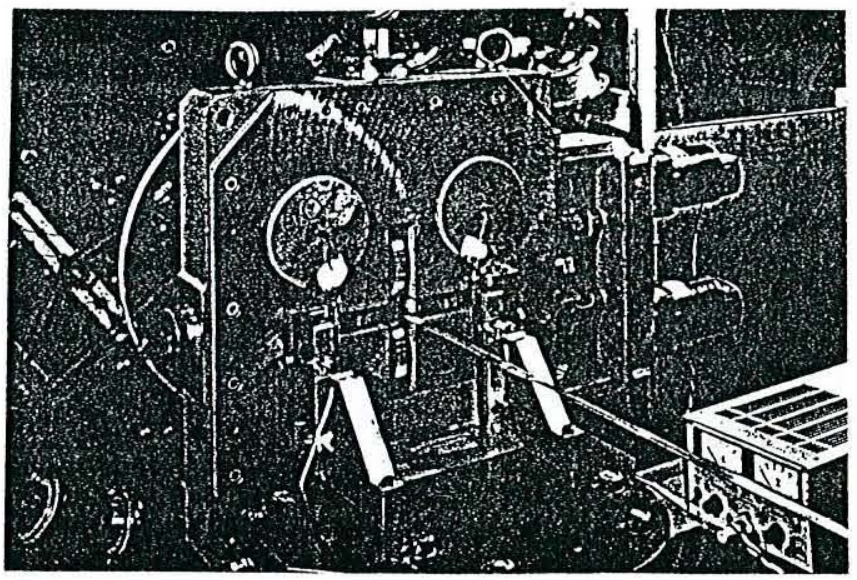

Fig. 1. Turkshead forming machine with top and bottom rollers powered in order to reduce cable tension.

However, the result may be to move the problem from cabling to the next step, i.e. coil winding, where tension must be applied in order to wind the cable at the coil ends. Indeed, the coil winding tests have indicated some definite directions for optimizing the cables.

The parameters that are important in coil winding are the following: (1)The cable edges must be rounded so that the insulation is not damaged. (2)The cable must be able to withstand the coil winding tension without collapsing. 
(3)The cable must withstand winding around the magnet ends without serious strand displacement (strand popping) or decabling problems. These parameters must be determined experimentally, so the cable samples were sent to FNAL where they were subjected to a series of winding tests.

Initial tests with bare inner cable were not promising. The cable has a tendency to collapse after exiting the end turn, and this could not be corrected by changing the cable tension. In addition, some strands tended to pop out of position as the cable exited the end turn. In summary, no combination of cable tension, winding direction, or pole piece shape produced a fully satisfactory winding with bare cable. The next series of tests were done with inner cable which was insulated with a half lap of $.025 \mathrm{~mm}$ thick $\times 9.5 \mathrm{~mm}$ wide polyimide, followed with a $.05 \mathrm{~mm} \times 9.5 \mathrm{~mm}$ wide polyimide film wound with a $2 \mathrm{~mm}$ gap (this is the insulation scheme proposed for the quadrupole magnet). The stability of the cable was improved significantly by the insulation, and no more cable collapsing was experienced. Although some strand popouts were still noted, these were readily corrected during the winding. Also, the shape of the end parts appeared to be the critical factor in this series of winding tests. When the angle of the pole was changed from 15 degrees (measured from vertical) to 8 degrees, both the strand popout tendency and the hump in the cable at the turn exit were reduced. Similar winding experiments were performed on both the 37 and the 38 strand insulated cables, and no difference in behavior was observed. In comparing coil winding in the favorable versus unfavorable direction, an increased tendency for strand popping was noted in the unfavorable direction.

Winding experiments turned next to the outer coils, where problems were anticipated due to the high aspect ratio of the outer cable. However, the results of coil winding tests with the insulated outer cable were surprisingly good. Winding tension, however, did play a role in the outer cable performance, with a tension of between $36-45 \mathrm{Kg}$ giving the best results. Once again, the pole angle was important, and better results were obtained when the pole angle was decreased from the initial 30 degrees to 16 degrees.

The winding tests showed that these high aspect cables could be wound around the small radius ends required for the LHC HGQ. However, it is desirable to wind both cables in their favorable direction. In order to accomplish this the wire twist direction will be changed for the inner layer so that the inner layer cable can be fabricated with the opposite lay direction. This is possible while still using the SSC wire, since the inventory contains a large quantity of inner layer wire that was stopped just before the wire twisting stage and so it can be twisted in the opposite direction.

The electrical tests on the cables also indicate some definite directions to take in order to optimize the design. Cable 563A (38 strand inner cable with a nominal width of $15.2 \mathrm{~mm}$ ) is definitely overcompacted, with a $33 \%$ degradation in critical current density. Both decreasing the number of strands (cable 563B) and increasing the cable width (cable 568) are effective in reducing the critical current degradation, and the critical current density in the cabled strands agree to better than $1 \%$ for these cables. However, a comparison of cable 568 with cable 569 indicates that the critical current density is still degraded about $4 \%$ in the 38 strand cable compared with the 37 strand cable. In other words, the potential increase in critical current due to adding one strand is canceled out by the degradation in the 38 strand cable. In order to fully optimize the inner cable with respect to critical current degradation, the width should be increased further, or the strand number should be reduced by one. However, before this final optimization is done, it is necessary to evaluate the other important properties, such as coil winding. In addition, the strand material may play a role in degradation, so additional cabling trials will be analyzed before a final configuration is established.

Samples of outer layer cable with 46 strands and with 45 strands were fabricated. However, the critical current was determined only for the 46 strand sample. The 45 strand sample was mechanically unstable; also, the critical current measurements on the 46 strand cable showed no degradation, hence, no need to reduce the compaction.

\section{CONCLUSIONS}

1. The high aspect layer cables required for a two-layer high gradient quadrupole can be fabricated without difficulty.

2. The outer layer cable has no problem with critical current degradation. The inner layer cable, however, has about 33\% Ic degradation when it is made with the initial parameters. This degradation is reduced to $4 \%$ when the cable is made in the 38 strand, $15.4 \mathrm{~mm}$ wide version. Eliminating one strand or increasing the cable width slightly should eliminate the remaining degradation.

3. Both inner and outer cables can be wound into the coils required for the quadrupole. However, the cables must be insulated and the coil end parts must be optimized in order to achieve good coil winding results. Both inner and outer cables should be wound in the favorable direction.

\section{ACKNOWLEDGMENTS}

We wish to thank Arup Ghosh, Bill Sampson, and their staff at BNL for measuring the critical currents on the cable samples. We also wish to acknowledge the contributions of the technical staff at FNAL for their help in the coil winding experiments. The work at LBNL is supported by the Director, Office of Energy Research, Office of High Energy and Nuclear Physics, High Energy Physics Division, U.S. Department of Energy, under Contract No. DE-AC03-76SF0098. FNAL is operated by Universities Research Association Inc., under contract with the U.S. Department of Energy.

\section{REFERENCES}

[1] R.C. Gupta et. al., "Design of a High Gradient Quadrupole for the LHC Interaction Regions" paper LN-6, these proceedings.

[2] R.M. Scanlan, "The Evolution of Tooling, Techniques, and Quality Control for Accelerator Dipole Magnet Cables," IEEE Trans. on Applied Superconductivity, 3 No. 1, March 1993, pp 842-849.

[3] H. Higley, "Calculations for Rutherford type Cabling," LBNL Internal Report \#LBID-2170, SC-Mag 560, 1995.

[4] M. Garber, A.K. Ghosh, and W.B. Sampson "The Effect of Self Field on the Critical Current Determination of Multifilamentary Superconductors," IEEE Trans. on Magnetics, 25, No. 2, March 1989, pp 1940-1944.

[5] J.M. Cook, "Strain Energy Minimization in SSC Magnet Winding", IEEE Trans. on Magnetics 27, No. 2, March 1991, pp 1976-1980. 